\title{
THE OPTIMUM ORIENTATION OF AN ABSORBING BARRIER
}

\author{
A D RAWLINS
}

\begin{abstract}
In the following work we solve the problem of the best orientation of a rigid noise barrier, which has one face lined with absorbent material, between a noise source and a receiver point in the shadow region of the barrier. By the best orientation is meant that positioning of the barrier that yields the least noise level at the receiving point for a given barrier and source position.
\end{abstract}

\section{INTRODUCTION}

In the following note we analyse the problem of optimizing the orientation of a noise barrier between a noise source and a receiver point. The noise barrier is assumed to be rigid on one side and to be lined with absorbent material on the other side. Such a barrier is more economic than a barrier lined on both sides with absorbent material. It also offers easier maintenance of the barrier. The question that arises is : How should one place such a barrier for a given source position to minimize the amplitude of the noise in the shadow region of the barrier where the receiver is placed?

In an earlier publication [1], numerical calculations for a limiting situation, where an infinite barrier, which was soft on one side and rigid on the other side, was insonified by a plane wave, led the author to suggest an optimum situation. However this was not proved rigorously, even for this somewhat hypothetical noise barrier. Here we shall consider a practical noise barrier where instead of a soft surface we have an absorbent surface, and we shall consider a point source.The assumption that the barrier is thin and infinite in length is maintained, since this is not an unreasonable assumption for the frequency of sound waves and the dimensions of barriers in practical situations. The only two situations we need to consider are shown in the figures 1 and 2, which we shall call case 1 and case 2 respectively. The question that now arises is which configuration, for a given fixed source and barrier position, gives the least noise level in the shadow region of the barrier? The shadow region is that region, on the opposite side of the barrier to the source of sound, where the sound source is not visible to the naked eye.

\section{The SOLUTiOn OF THE ABSORBING HALF PLANE PROBLEM}

It can be shown that for small perturbations of a compressible fluid from rest, the pressure variation $p$, density fluctuation $\rho$, and velocity potential $\phi$, satisfy a scalar wave equation

$$
\left(\nabla^{2}-c^{-2} \partial^{2} / \partial t^{2}\right) p(\mathbf{x}, t)=q(\mathbf{x}, t)
$$

Date: July 10, 2003 and, in revised form, July 30, 2004,March 2, 2005

1991 Mathematics Subject Classification. Primary 47A15; Secondary 46A32, 47D20.

Key words and phrases. Noise Barrier, Diffraction, Wave Scattering. 


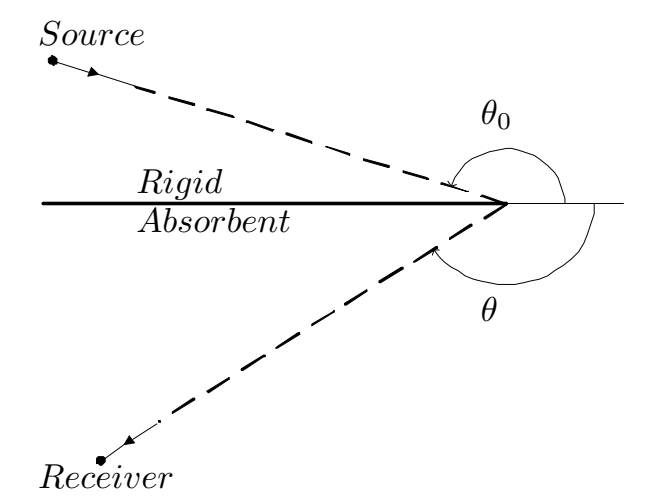

Figure 1. A source and receiver separated by a noise barrier,Case 1.

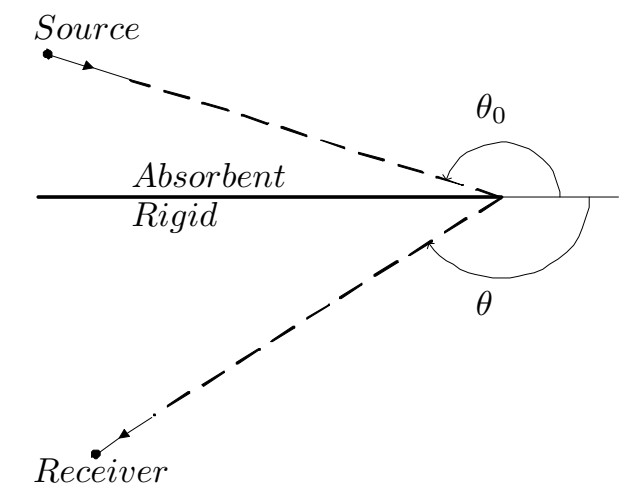

FiguRE 2. A source and receiver separated by a noise barrier,Case 2 .

where $c$ is the wave speed and $q$ represents a distribution of sounds sources. The three variables $p, \rho$, and $\phi$, are related through the formulae

$$
p=c^{-2} \rho=-\rho_{0} \partial \phi / \partial t,
$$

where $\rho_{0}$ is the mean fluid density.

If the forcing term $q(\mathbf{x}, t)$ and the boundary conditions are simple harmonic in time, with angular frequency $\omega$, then the linear nature of (1) ensures that $p$ will have similar time dependance. Thus if 


$$
q(\mathbf{x}, t)=\Re\left(q(\mathbf{x}) e^{-i \omega t}\right), p(\mathbf{x}, t)=\Re\left(p(\mathbf{x}) e^{-i \omega t}\right),
$$

and the wave equation (1) becomes the Helmholtz equation

$$
\left(\nabla^{2}+k^{2}\right) p(\mathbf{x})=q(\mathbf{x})
$$

where $k=\omega / c$ is the acoustic wavenumber. We shall assume that the compressible fluid occupies the region $r>0,-\pi<\theta<\pi,-\infty<z<\infty$, where $(r, \theta)$ are polar coordinates in the $(x, y)$-plane, related to the Cartesian variables $(x, y)$ by $x=r \cos \theta, y=r \sin \theta$.

We shall also assume that the pressure sound source is a harmonic cylindrical line radiator located at $r=r_{0}$ and $\theta=\theta_{0}$ and parallel with the edge of the thin half plane, see figure 3 . Then, the two-dimensional pressure wave field $p(r, \theta)$ satisfies the following boundary value problem:

In the region $r>0,-\pi<\theta<\pi,-\infty<z<\infty$, it satisfies the reduced wave equation

$$
\left(\frac{\partial^{2}}{\partial r^{2}}+\frac{1}{r} \frac{\partial}{\partial r}+\frac{1}{r^{2}} \frac{\partial^{2}}{\partial \theta^{2}}+k^{2}\right) p(r, \theta)=\frac{\delta\left(r-r_{0}\right) \delta\left(\theta-\theta_{0}\right)}{r_{0}}
$$

On the faces of the half plane the field satisfies the boundary conditions

$$
\frac{1}{r} \frac{\partial p}{\partial \theta} \mp i k \sin \vartheta_{ \pm} p=0, \quad(\theta= \pm \pi)
$$

The complex angles $\vartheta_{ \pm}$are related to normalized surface impedances of the barrier faces (the actual surface impedances are $Z_{ \pm}=\rho c / \sin \theta_{ \pm}, \rho c$ being the acoustic impedance, so that $\sin \vartheta_{ \pm}$are really the normalized surface admittances). For any physically passive boundary, which is free of field sources, simple energy considerations require that $0<\Re \vartheta_{ \pm} \leq \pi / 2$, whereas $\Im \vartheta_{ \pm}$can be arbitrary. The physical problem has a unique solution and this requires that the mathematical problem should satisfy certain energy conditions at the edge $(r=0)$ and far from the edge $(r \rightarrow \infty)$ uniformly in $\theta$.

The edge condition requires that

$$
\lim _{r \rightarrow 0}|p(r, \theta)|=\text { const. } \lim _{r \rightarrow 0}|r \nabla p(r, \theta)|=0 .
$$

This ensures that the edge does not radiate energy and only passively radiates cylindrical waves from the edge.

The extinction condition requires that

$$
\lim _{r \rightarrow \infty}|p(r, \theta)|=0, \quad \Im k>0 .
$$

This condition at infinity is more generally applicable and analytically more convenient than the commonly used radiation condition. The requirement $\Im k>0$ implies that the medium has some absorption, which can be arbitrary small. Thus all waves at infinity must be evanescent and no energy is transported to the infinite regions.

The only source of energy in this problem is the delta-source in the right hand side of equation (2) which produces cylindrical waves described by the free space Green function $\frac{1}{4 i} H_{0}^{(1)}(k R)$ where $R=\sqrt{ }\left(r^{2}+r_{0}^{2}-2 r r_{0} \cos \left(\theta-\theta_{0}\right)\right)$. Without loss of generality we shall assume that $\pi / 2<\theta_{0}<\pi$. The corresponding shadow region of the barrier is defined as $-\pi<\theta<\theta_{0}-\pi$. 


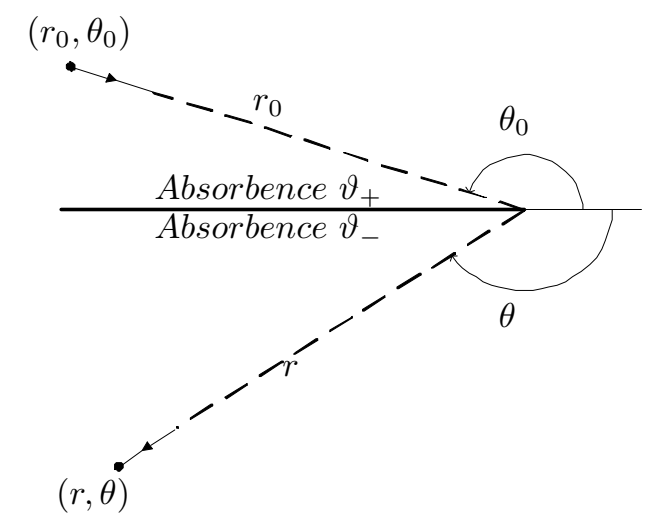

FIGURE 3. Diffraction of a cylindrical sound wave by an absorbent barrier.

The exact solution of the problem of the diffraction of the waves from line source by an absorbing half plane and a wedge with different absorbent linings on the two faces of the half plane and the wedge have been given by [2] and [3] respectively.The geometry of the half plane problem is shown in figure 3.

For a distance of a couple of wavelengths from the edge of the half plane the exact solution, which consists of a complex integral, is accurately represented by its asymptotic form. In particular it can be shown that the field in the shadow region of the half plane is given by

$$
p(r, \theta)=\frac{i D\left(\theta, \theta_{0}\right)}{4 \pi k \sqrt{r r_{0}}} e^{i k\left(r+r_{0}\right)}\left[1+O\left(\frac{1}{k r_{<}}\right)\right]
$$

with $r_{<}=\min \left(r, r_{0}\right)$.

The function

$$
D\left(\theta, \theta_{0}\right)=T\left(\theta, \theta_{0}\right) L\left(\theta, \theta_{0}\right) /\left(\Psi(\theta) \Psi\left(\theta_{0}\right)\right),
$$

where the function $T\left(\theta, \theta_{0}\right)$ is independent of the impedance parameters $\vartheta_{ \pm}$, and

$$
L\left(\theta, \theta_{0}\right)=\left[2 \sin \frac{1}{2} \theta \sin \frac{1}{2} \theta_{0}+1-2 q_{+} q_{-}-\sqrt{2}\left(\sin \frac{1}{2} \theta+\sin \frac{1}{2} \theta_{0}\right)\left(q_{+}-q_{-}\right)\right],
$$

with $q_{ \pm}=\cos \left[\frac{1}{4}\left(\pi-2 \vartheta_{ \pm}\right)\right]$; and

$\Psi(\theta)=\psi_{\pi}\left(\theta+3 \pi / 2-\vartheta_{+}\right) \psi_{\pi}\left(\theta+\pi / 2+\vartheta_{+}\right) \psi_{\pi}\left(\theta-\pi / 2-\vartheta_{-}\right) \psi_{\pi}\left(\theta-3 \pi / 2+\vartheta_{-}\right)$,

where the function $\psi_{\pi}(\alpha)$ is a special function introduced by [4], from where the following properties can be deduced.

$$
\begin{gathered}
\psi_{\pi}(\alpha)=\exp \left[-\frac{1}{8 \pi} \int_{0}^{\alpha} \frac{\pi \sin u-2 \sqrt{2} \pi \sin \frac{1}{2} u+2 u}{\cos u} d u\right] \\
\psi_{\pi}(\alpha+\pi / 2) \psi_{\pi}(\alpha-\pi / 2)=\psi_{\pi}^{2}(\pi / 2) \cos \alpha / 4
\end{gathered}
$$


From the above results it can be shown that

$$
\frac{\psi_{\pi}(\alpha+\pi)}{\psi_{\pi}(\alpha-\pi)}=\frac{\cos \frac{1}{4}(\alpha+\pi / 2)}{\cos \frac{1}{4}(\alpha-\pi / 2)},
$$

and

$$
\begin{aligned}
\Psi(\alpha) \Psi(\alpha-\pi) & =\text { const. } \cos \frac{1}{4}\left(\alpha+\pi-\vartheta_{+}\right) \cos \frac{1}{4}\left(\alpha+\vartheta_{+}\right) \\
& \times \cos \frac{1}{4}\left(\alpha-\pi-\vartheta_{-}\right) \cos \frac{1}{4}\left(\alpha-2 \pi+\vartheta_{-}\right),
\end{aligned}
$$

where the constant is independent of $\alpha$, and $\vartheta_{ \pm}$.

\section{THE COMPARISON OF THE TWO ABSORBING HALF PLANE PROBLEMS}

We shall now use the above results, related to figure 3 , to give the field representation for the pressure magnitude in the shadow region for the two barrier positions shown in figure 1 and figure 2. For the situation shown in figure 1 we choose $\vartheta_{+}=0(\theta=\pi)$, and $\vartheta_{-}=\vartheta(\theta=-\pi)$; whereas for the situation shown in figure 2 we choose $\vartheta_{+}=\vartheta(\theta=\pi)$, and $\vartheta_{-}=0(\theta=-\pi)$, where $0<\Re \vartheta \leq \pi / 2$. Then by using the appropriate suffixes 1 and 2 for both these situations we have

$$
\begin{aligned}
& p_{1}(r, \theta)=\frac{i D_{1}\left(\theta, \theta_{0}\right)}{4 \pi k \sqrt{r r_{0}}} e^{i k\left(r+r_{0}\right)}\left[1+O\left(\frac{1}{k r_{<}}\right)\right],\left(-\pi<\theta<\theta_{0}-\pi\right), \\
& p_{2}(r, \theta)=\frac{i D_{2}\left(\theta, \theta_{0}\right)}{4 \pi k \sqrt{r r_{0}}} e^{i k\left(r+r_{0}\right)}\left[1+O\left(\frac{1}{k r_{<}}\right)\right],\left(-\pi<\theta<\theta_{0}-\pi\right),
\end{aligned}
$$

where

$$
\begin{aligned}
& D_{1}\left(\theta, \theta_{0}\right)=T\left(\theta, \theta_{0}\right) L_{1}\left(\theta, \theta_{0}\right) /\left(\Psi_{1}(\theta) \Psi_{1}\left(\theta_{0}\right)\right), \\
& D_{2}\left(\theta, \theta_{0}\right)=T\left(\theta, \theta_{0}\right) L_{2}\left(\theta, \theta_{0}\right) /\left(\Psi_{2}(\theta) \Psi_{2}\left(\theta_{0}\right)\right),
\end{aligned}
$$

with

$$
\begin{gathered}
\Psi_{1}(\theta)=\psi_{\pi}(\theta+3 \pi / 2) \psi_{\pi}(\theta+\pi / 2) \psi_{\pi}(\theta-\pi / 2-\vartheta) \psi_{\pi}(\theta-3 \pi / 2+\vartheta), \\
\Psi_{2}(\theta)=\psi_{\pi}(\theta+3 \pi / 2-\vartheta) \psi_{\pi}(\theta+\pi / 2+\vartheta) \psi_{\pi}(\theta-\pi / 2) \psi_{\pi}(\theta-3 \pi / 2), \\
L_{1}\left(\theta, \theta_{0}\right)=2 \sin \frac{1}{2} \theta \sin \frac{1}{2} \theta_{0}+\left(\sin \frac{1}{2} \theta+\sin \frac{1}{2} \theta_{0}-1\right)(\sqrt{2} q-1), \\
L_{2}\left(\theta, \theta_{0}\right)=2 \sin \frac{1}{2} \theta \sin \frac{1}{2} \theta_{0}-\left(\sin \frac{1}{2} \theta+\sin \frac{1}{2} \theta_{0}+1\right)(\sqrt{2} q-1) .
\end{gathered}
$$

In the last two expressions $q=\cos \frac{1}{4}(\pi-2 \vartheta)$, and since $0<\Re \vartheta \leq \pi / 2$, then

$$
0<\Re(\sqrt{2} q-1)
$$


We shall now prove the following theorem:

\section{Theorem}

For all $-\pi \leq \theta \leq \theta_{0}-\pi, \pi / 2 \leq \theta_{0} \leq \pi$, then

$$
\begin{aligned}
& 0 \leq\left|p_{1}(r, \theta) / p_{2}(r, \theta)\right| \leq R_{p}\left(\theta, \theta_{0}\right)<R_{\infty}\left(\theta, \theta_{0}\right)<1,\left(-\pi \leq \theta \leq-\theta_{0}\right) \\
& 1 \leq\left|p_{1}(r, \theta) / p_{2}(r, \theta)\right| \leq R_{\infty}\left(\theta, \theta_{0}\right)<R_{p}\left(\theta, \theta_{0}\right)<\infty,\left(-\theta_{0} \leq \theta \leq \theta_{0}-\pi\right) .
\end{aligned}
$$

$R_{p}\left(\theta, \theta_{0}\right)$ is the root of a specific quartic equation; and

$$
R_{\infty}\left(\theta, \theta_{0}\right)=\frac{\left(1-\sin \frac{1}{2} \theta-\sin \frac{1}{2} \theta_{0}\right) \cos \frac{1}{4}(\theta-\pi) \cos \frac{1}{4}\left(\theta_{0}-\pi\right)}{\left(1+\sin \frac{1}{2} \theta+\sin \frac{1}{2} \theta_{0}\right) \cos \frac{1}{4}(\theta+\pi) \cos \frac{1}{4}\left(\theta_{0}+\pi\right)} \leq R_{\infty}\left(\theta_{\infty}, \theta_{0}\right)
$$

where

\section{Proof}

If we call the ratio

$$
\theta_{\infty}=2 \arcsin \left[\sin \frac{1}{2} \theta_{0}-\sqrt{2-\cos \theta_{0}}\right]
$$

$$
R\left(\theta, \theta_{0}\right)=\left|p_{1}(r, \theta) / p_{2}(r, \theta)\right|,
$$

then from equations (5) and(6) and the above results we have

$$
R\left(\theta, \theta_{0}\right)=\left|\frac{L_{1}\left(\theta, \theta_{0}\right)}{L_{2}\left(\theta, \theta_{0}\right)} \frac{\Psi_{2}(\theta) \Psi_{2}\left(\theta_{0}\right)}{\Psi_{1}(\theta) \Psi_{1}\left(\theta_{0}\right)}\right|
$$

with

$$
\left|\frac{L_{1}\left(\theta, \theta_{0}\right)}{L_{2}\left(\theta, \theta_{0}\right)}\right|=\left|\frac{2 \sin \frac{1}{2} \theta \sin \frac{1}{2} \theta_{0}+\left(\sin \frac{1}{2} \theta+\sin \frac{1}{2} \theta_{0}-1\right)(\sqrt{2} q-1)}{2 \sin \frac{1}{2} \theta \sin \frac{1}{2} \theta_{0}-\left(\sin \frac{1}{2} \theta+\sin \frac{1}{2} \theta_{0}+1\right)(\sqrt{2} q-1)}\right|,
$$

and

$$
\frac{\Psi_{2}(\theta)}{\Psi_{1}(\theta)}=\left[\frac{\psi_{\pi}(\theta+3 \pi / 2-\vartheta)}{\psi_{\pi}(\theta-\pi / 2-\vartheta)}\right]\left[\frac{\psi_{\pi}(\theta+\pi / 2+\vartheta)}{\psi_{\pi}(\theta-3 \pi / 2+\vartheta)}\right]\left[\frac{\psi_{\pi}(\theta-\pi / 2)}{\psi_{\pi}(\theta+3 \pi / 2)}\right]\left[\frac{\psi_{\pi}(\theta-3 \pi / 2)}{\psi_{\pi}(\theta+\pi / 2)}\right]
$$

By using the result (3) the above expression reduces to the purely trigonometric form

$$
\frac{\Psi_{2}(\theta)}{\Psi_{1}(\theta)}=\left[\frac{\cos \left(\frac{1}{4}(\theta-\vartheta+\pi)\right)}{\cos \left(\frac{1}{4}(\theta-\vartheta)\right)}\right]\left[\frac{\cos \left(\frac{1}{4}(\theta+\vartheta)\right)}{\cos \left(\frac{1}{4}(\theta+\vartheta-\pi)\right)}\right]\left[\frac{\cos \left(\frac{1}{4} \theta\right)}{\cos \left(\frac{1}{4}(\theta+\pi)\right)}\right]\left[\frac{\cos \left(\frac{1}{4}(\theta-\pi)\right)}{\cos \left(\frac{1}{4} \theta\right)}\right]
$$

or

$$
\frac{\Psi_{2}(\theta)}{\Psi_{1}(\theta)}=\left[\frac{\cos \left(\frac{1}{4}(\theta-\vartheta+\pi)\right)}{\cos \left(\frac{1}{4}(\theta-\vartheta)\right)}\right]\left[\frac{\cos \left(\frac{1}{4}(\theta+\vartheta)\right)}{\cos \left(\frac{1}{4}(\theta+\vartheta-\pi)\right)}\right]\left[\frac{\cos \left(\frac{1}{4}(\theta-\pi)\right.}{\cos \left(\frac{1}{4}(\theta+\pi)\right)}\right] .
$$

After a bit of trigonometry this can be rewritten as

$$
=\left[\frac{\sqrt{2} \cos \frac{1}{2} \theta \cos \frac{1}{4} \theta+(\sqrt{2} q-1) \cos \frac{1}{4}(\theta-\pi)}{\sqrt{2} \cos \frac{1}{2} \theta \cos \frac{1}{4} \theta+(\sqrt{2} q-1) \cos \frac{1}{4}(\theta+\pi)}\right] .
$$

Thus substituting the results (8) and (9) into (7) we have 


$$
\begin{aligned}
R\left(\theta, \theta_{0}\right)= & \left|\frac{2 \sin \frac{1}{2} \theta \sin \frac{1}{2} \theta_{0}+\left(\sin \frac{1}{2} \theta+\sin \frac{1}{2} \theta_{0}-1\right)(\sqrt{2} q-1)}{2 \sin \frac{1}{2} \theta \sin \frac{1}{2} \theta_{0}-\left(\sin \frac{1}{2} \theta+\sin \frac{1}{2} \theta_{0}+1\right)(\sqrt{2} q-1)}\right| \\
& \times\left|\frac{\sqrt{2} \cos \frac{1}{2} \theta \cos \frac{1}{4} \theta+(\sqrt{2} q-1) \cos \frac{1}{4}(\theta-\pi)}{\sqrt{2} \cos \frac{1}{2} \theta \cos \frac{1}{4} \theta+(\sqrt{2} q-1) \cos \frac{1}{4}(\theta+\pi)}\right| \\
& \times\left|\frac{\sqrt{2} \cos \frac{1}{2} \theta_{0} \cos \frac{1}{4} \theta_{0}+(\sqrt{2} q-1) \cos \frac{1}{4}\left(\theta_{0}-\pi\right)}{\sqrt{2} \cos \frac{1}{2} \theta_{0} \cos \frac{1}{4} \theta_{0}+(\sqrt{2} q-1) \cos \frac{1}{4}\left(\theta_{0}+\pi\right)}\right| .
\end{aligned}
$$

We can now see by inspection that $R\left(-\theta_{0}, \theta_{0}\right)=1$ which is a consequence of the reciprocity theorem. We can also see that $R\left(-\pi, \theta_{0}\right)=0$ which is a consequence of the Karp-Karal lemma, (see [5], and the appendix A) that for an absorbing boundary condition the far field on the shadow side of a half plane must vanish, whereas for a rigid boundary condition the far field on the shadow side of the half plane is a non zero constant. We also note from the results (3) and (4) that

$$
\frac{\Psi_{2}\left(\theta_{0}\right) \Psi_{2}\left(\theta_{0}-\pi\right)}{\Psi_{1}\left(\theta_{0}\right) \Psi_{1}\left(\theta_{0}-\pi\right)}=\frac{\cos \frac{1}{4}\left(\theta_{0}+\pi-\vartheta\right) \cos \frac{1}{4}\left(\theta_{0}+\vartheta\right) \cos \frac{1}{4}\left(\theta_{0}-\pi\right) \sin \frac{1}{4} \theta_{0}}{\cos \frac{1}{4}\left(\theta_{0}+\pi\right) \cos \frac{1}{4} \theta_{0} \cos \frac{1}{4}\left(\theta_{0}-\pi-\vartheta\right) \sin \frac{1}{4}\left(\theta_{0}+\vartheta\right)},
$$

and since

$$
\begin{aligned}
\frac{L_{1}\left(\theta_{0}-\pi, \theta_{0}\right)}{L_{2}\left(\theta_{0}-\pi, \theta_{0}\right)} & =\frac{-2 \cos \frac{1}{2} \theta_{0} \sin \frac{1}{2} \theta_{0}+\left[1+\cos \frac{1}{2} \theta_{0}-\sin \frac{1}{2} \theta_{0}\right](1-\sqrt{2} q)}{-2 \cos \frac{1}{2} \theta_{0} \sin \frac{1}{2} \theta_{0}+\left[1-\cos \frac{1}{2} \theta_{0}+\sin \frac{1}{2} \theta_{0}\right](1-\sqrt{2} q)} \\
& =\frac{\cos \frac{1}{4} \theta_{0} \cos \frac{1}{4}\left(\theta_{0}+\pi\right)}{\sin \frac{1}{4} \theta_{0} \cos \frac{1}{4}\left(\theta_{0}-\pi\right)} \frac{\sin \frac{1}{4}\left(\theta_{0}-\vartheta+\pi\right) \sin \frac{1}{4}\left(\theta_{0}+\vartheta\right)}{\left.\cos \frac{1}{4}\left(\theta_{0}+\vartheta\right) \cos \frac{1}{4}\left(\theta_{0}-\vartheta+\pi\right)\right]}
\end{aligned}
$$

then on combining the above two results into the ratio we see that

$$
R\left(\theta_{0}-\pi, \theta_{0}\right)=\left|\frac{L_{1}\left(\theta_{0}-\pi, \theta_{0}\right)}{L_{2}\left(\theta_{0}-\pi, \theta_{0}\right)} \frac{\Psi_{2}\left(\theta_{0}\right) \Psi_{2}\left(\theta_{0}-\pi\right)}{\Psi_{1}\left(\theta_{0}\right) \Psi_{1}\left(\theta_{0}-\pi\right)}\right|=1 .
$$

Strictly speaking the ray $\theta=\theta_{0}-\pi$ is not in the shadow region of the half planes but on the boundary between the insonified and shadow region. The far field diffraction coefficient for each problem becomes infinite on this boundary. However the total field is continuous and therefore the ratio in the limit as $\theta \longrightarrow \theta_{0}-\pi$ is correct. It is a surprising and interesting fact, that although the two problems are different, on this boundary the field is the same!

In order to prove the theorem, and avoid long formulae, we make the following abbreviations. The expression (10) is rewritten as

$$
R\left(\theta, \theta_{0}\right)=|F(z)|, \quad(\Re z>0) ;
$$

where

$$
F(z)=\frac{(a+b z)(c+d z)(e+f z)}{\left(a+b^{\prime} z\right)\left(c+d^{\prime} z\right)\left(e+f^{\prime} z\right)}
$$

and

$$
\begin{gathered}
z=\sqrt{2} q-1, \\
a=2 \sin \frac{1}{2} \theta \sin \frac{1}{2} \theta_{0},
\end{gathered}
$$




$$
\begin{gathered}
b=\sin \frac{1}{2} \theta+\sin \frac{1}{2} \theta_{0}-1, \\
b^{\prime}=-\sin \frac{1}{2} \theta-\sin \frac{1}{2} \theta_{0}-1, \\
c=\sqrt{2} \cos \frac{1}{2} \theta \cos \frac{1}{4} \theta \\
d=\cos \frac{1}{4}(\theta-\pi) \\
d^{\prime}=\cos \frac{1}{4}(\theta+\pi), \\
e=\sqrt{2} \cos \frac{1}{2} \theta_{0} \cos \frac{1}{4} \theta_{0} \\
f=\cos \frac{1}{4}\left(\theta_{0}-\pi\right), \\
f^{\prime}=\cos \frac{1}{4}\left(\theta_{0}+\pi\right) .
\end{gathered}
$$

It is not difficult to show that all the zeros and poles of $F(z)$ lie in the region $\Re z \leq$ 0 . The function $F(z)$ is therefore holomorphic in $\Re z>0$. We shall now apply the maximum modulus theorem to the function $F(z)$ on the infinite semi-circle in $\Re z \geq$ 0 , defined by $(z=0+\imath y,-\varrho<y<\varrho) \cup(z=\varrho \exp \imath \varphi,-\pi / 2<\varphi<\pi / 2), \varrho \rightarrow \infty$.

On the large semi-circular region $|z|=\varrho \rightarrow \infty,-\pi / 2<\varphi<\pi / 2$,

$$
\begin{aligned}
|F(z)| & =\left|\frac{b d f}{b^{\prime} d^{\prime} f^{\prime}}\right|=\frac{\left(1-\sin \frac{1}{2} \theta-\sin \frac{1}{2} \theta_{0}\right) \cos \frac{1}{4}(\theta-\pi) \cos \frac{1}{4}\left(\theta_{0}-\pi\right)}{\left(1+\sin \frac{1}{2} \theta+\sin \frac{1}{2} \theta_{0}\right) \cos \frac{1}{4}(\theta+\pi) \cos \frac{1}{4}\left(\theta_{0}+\pi\right)} \\
& <1 \quad\left(-\pi<\theta<-\theta_{0}\right) \\
& >1 \quad\left(-\theta_{0}<\theta<\theta_{0}-\pi\right) .
\end{aligned}
$$

On the imaginary axes $z=\imath y,-\infty<y<\infty$, we have

$$
|F(\iota y)|^{2}=\left(\frac{a^{2}+b^{2} y^{2}}{a^{2}+b^{\prime 2} y^{2}}\right)\left(\frac{c^{2}+d^{2} y^{2}}{c^{2}+d^{\prime 2} y^{2}}\right)\left(\frac{e^{2}+f^{2} y^{2}}{e^{2}+f^{\prime 2} y^{2}}\right) .
$$

The determination of the maximum and minimum values of this real rational polynomial whose numerator and denominator is a real cubic in $y^{2}$ can be reduced to the solution of a quartic equation in $y^{2}$, see appendix B. The nature and relative magnitude of these extrema requires further analysis. By considering the function $F_{1}(y)=|F(\iota y)|^{2}-\left|\frac{b d f}{b^{\prime} d^{\prime} f^{\prime}}\right|^{2}=0$, then it can be shown that the zeros of $F_{1}(y)=0$ are the roots of a quadratic equation in $y^{2}$. Since $|F(0)|^{2}=1$; and as $y \rightarrow \pm \infty$, $|F(\iota y)|^{2} \rightarrow\left|\frac{b d f}{b^{\prime} d^{\prime} f^{\prime}}\right|^{2}$ from below for $\left(-\pi<\theta<-\theta_{0}\right)$ and from above for $\left(-\theta_{0}<\right.$ $\left.\theta<\theta_{0}-\pi\right)$ then since $|F(\iota y)|^{2}$ is a continuous function of $y$ only one turning point occurs at a finite positive value $y_{p}$ where $0<y_{p}<\infty$.

By taking the logarithmic derivative of the last expression for $|F(\iota y)|^{2}$ we get 


$$
\begin{aligned}
\frac{d|F(\iota y)|}{d y}= & y|F(\iota y)|\left(\frac{1}{(a / b)^{2}+y^{2}}+\frac{1}{(c / d)^{2}+y^{2}}+\frac{1}{(e / f)^{2}+y^{2}}\right. \\
& \left.-\frac{1}{\left(a / b^{\prime}\right)^{2}+y^{2}}-\frac{1}{\left(c / d^{\prime}\right)^{2}+y^{2}}-\frac{1}{\left(e / f^{\prime}\right)^{2}+y^{2}}\right) \\
\equiv & y|F(\iota y)|\left(A_{0}+A_{1} y^{2}+A_{2} y^{4}+A_{3} y^{6}+A_{4} y^{8}\right) /\left[\left((a / b)^{2}+y^{2}\right)\right. \\
& \left.\left((c / d)^{2}+y^{2}\right)\left((e / f)^{2}+y^{2}\right)\left(\left(a / b^{\prime}\right)^{2}+y^{2}\right)\left(\left(c / d^{\prime}\right)^{2}+y^{2}\right)\left(\left(e / f^{\prime}\right)^{2}+y^{2}\right)\right] .
\end{aligned}
$$

Now $d|F(\iota y)| / d y=0$, at $y=0, y= \pm y_{p}$, and as $y \rightarrow \pm \infty$. These are the only places where $d|F(\iota y)| / d y$ vanishes. The point $y_{p}$ is determined explicitly by solving the quartic equation $A_{0}+A_{1} y^{2}+A_{2} y^{4}+A_{3} y^{6}+A_{4} y^{8}$, in $y^{2}$. This latter equation only has one positive root which corresponds to $y= \pm y_{p}$. Although the equation can be solved explicitly by means of Mathematica it is very complicated. In practice the appropriate positive solution can be obtained by substituting a set of numerical values into the equation to identify the positive root and then invoke the fact that the roots of a polynomial equation are continuous functions of the coefficients to identify the appropriate mathematical expression for the root $y_{p}$. Corresponding to this root will be an extremum, say, $R_{p}\left(\theta, \theta_{0}\right)=\left|F\left(\iota y_{p}\right)\right|^{2}$. Since $|F(\iota y)|^{2}$ is a continuous function of $y$, the nature of the extreme values at the turning points $y=0, y= \pm y_{p}, y \rightarrow \pm \infty$ are determined by finding the nature of the extremum at one turning point, say $y=0$. This nature is determined by the sign of the function

$$
S=\left(\left(\frac{b}{a}\right)^{2}+\left(\frac{d}{c}\right)^{2}+\left(\frac{f}{e}\right)^{2}-\left(\frac{b^{\prime}}{a}\right)^{2}-\left(\frac{d^{\prime}}{c}\right)^{2}-\left(\frac{f^{\prime}}{e}\right)^{2}\right),
$$

since the sign of the function

$$
\begin{array}{r}
\left(\frac{1}{(a / b)^{2}+y^{2}}+\frac{1}{(c / d)^{2}+y^{2}}+\frac{1}{(e / f)^{2}+y^{2}}\right. \\
\left.-\frac{1}{\left(a / b^{\prime}\right)^{2}+y^{2}}-\frac{1}{\left(c / d^{\prime}\right)^{2}+y^{2}}-\frac{1}{\left(e / f^{\prime}\right)^{2}+y^{2}}\right),
\end{array}
$$

does not change in the neighbourhood of $y=0$, for $-\epsilon \leq y \leq \epsilon, \epsilon>0, \epsilon<<1$. By using Mathematica it can be shown that

$$
\begin{aligned}
S= & {\left[\csc \frac{1}{4} \theta_{0} \csc \frac{1}{4} \theta \sec \frac{1}{4} \theta_{0} \sec \frac{1}{4} \theta\right]^{2}\left[\cos \frac{1}{2}\left(\theta_{0}-\theta\right) \sin \frac{1}{4}\left(\theta_{0}+\theta\right)\right] } \\
& {\left[2 \cos \frac{1}{4}\left(\theta_{0}-5 \theta\right)-\cos \frac{1}{4}\left(3 \theta_{0}-5 \theta\right)-\cos \frac{1}{4}\left(5 \theta_{0}-3 \theta\right)-4 \cos \frac{1}{4}\left(\theta_{0}-\theta\right)\right.} \\
& +2 \cos \frac{1}{4}\left(5 \theta_{0}-\theta\right)+\cos \frac{3}{4}\left(\theta_{0}+\theta\right)+\cos \frac{5}{4}\left(\theta_{0}+\theta\right)-6 \cos \frac{1}{4}\left(3 \theta_{0}+\theta\right) \\
& \left.-6 \cos \frac{1}{4}\left(\theta_{0}+3 \theta\right)-2 \cos \frac{1}{4}\left(5 \theta_{0}+3 \theta\right)-2 \cos \frac{1}{4}\left(3 \theta_{0}+5 \theta\right)\right] / \\
& {\left[128\left(\cos \frac{\theta_{0}}{4}+\sin \frac{\theta_{0}}{4}\right)^{2}\left(1-\sin \frac{\theta_{0}}{2}\right)\left(\cos \frac{\theta}{4}+\sin \frac{\theta}{4}\right)^{2}\left(1-\sin \frac{\theta}{2}\right)\right], }
\end{aligned}
$$

clearly the sign of $\mathrm{S}$ is given for the range of interest of $-\pi<\theta<\theta_{0}-\pi$ and $\pi / 2<\theta_{0}<\pi$ by the sign of the expression :

$$
\begin{aligned}
{\left[\cos \frac{1}{2}\left(\theta_{0}-\theta\right) \sin \frac{1}{4}\left(\theta_{0}+\theta\right)\right] } & >0 \quad\left(-\pi<\theta<-\theta_{0}\right), \\
& <0 \quad\left(-\theta_{0}<\theta<\theta_{0}-\pi\right) ;
\end{aligned}
$$


and the sign of the expression:

$$
\begin{aligned}
G= & \frac{1}{16}\left[2 \cos \frac{1}{4}\left(\theta_{0}-5 \theta\right)-\cos \frac{1}{4}\left(3 \theta_{0}-5 \theta\right)-\cos \frac{1}{4}\left(5 \theta_{0}-3 \theta\right)-4 \cos \frac{1}{4}\left(\theta_{0}-\theta\right)\right. \\
& +2 \cos \frac{1}{4}\left(5 \theta_{0}-\theta\right)+\cos \frac{3}{4}\left(\theta_{0}+\theta\right)+\cos \frac{5}{4}\left(\theta_{0}+\theta\right)-6 \cos \frac{1}{4}\left(3 \theta_{0}+\theta\right) \\
& \left.-6 \cos \frac{1}{4}\left(\theta_{0}+3 \theta\right)-2 \cos \frac{1}{4}\left(5 \theta_{0}+3 \theta\right)-2 \cos \frac{1}{4}\left(3 \theta_{0}+5 \theta\right)\right] .
\end{aligned}
$$

The latter expression can be put in the form of the trigonometric polynomial:

$$
G(\xi, \eta)=-\xi^{3}+\xi^{5}+\eta-\xi^{2} \eta-2 \xi^{4} \eta+\xi \eta^{2}-\eta^{3}+2 \xi^{2} \eta^{3}-\xi \eta^{4},
$$

where $\xi=\cos \frac{\theta+\theta_{0}}{4}, \eta=\cos \frac{\theta-\theta_{0}}{4}$.

Clearly for $-\pi<\theta<\theta_{0}-\pi$ then

$$
1 / \sqrt{2}<\xi<1,0<\eta<\frac{1}{2} \sqrt{2+\sqrt{2}}
$$

By using Mathematica a plot of the curves of $G(\xi, \eta)=0$ can be displayed, see figure 3. In figure 3 the horizontal axis is $\xi$ and the vertical axis is $\eta$. In the regions between these curves $G(\xi, \eta)>0$ or $G(\xi, \eta)<0$. It can also be shown by using Mathematica that:

$$
\begin{gathered}
\operatorname{Resultant}\left[G(\xi, \eta), G_{\xi}(\xi, \eta)\right]= \\
-4\left(1-\eta^{2}\right) \eta^{2}\left(-27-513 \eta^{2}-1885 \eta^{4}+7216 \eta^{6}-7208 \eta^{8}+1936 \eta^{10}+432 \eta^{12}\right),
\end{gathered}
$$

and

$\operatorname{Resultant}\left[G(\xi, \eta), G_{\eta}(\xi, \eta)\right]=4 \xi\left(1-2 \xi^{2}\right)^{2}\left(-1+7 \xi^{2}-2 \xi^{4}-\xi^{6}-52 \xi^{8}+108 \xi^{10}\right)$

From the last two results the horizontal and vertical tangents, respectively, to the curves can be calculated exactly from the roots of the factored equations. It is then easily seen from the figure that for $-\pi<\theta<\theta_{0}-\pi$,

$$
G<0 \text {. }
$$

Hence $S<0$ for $-\pi<\theta<-\theta_{0}$; and $S>0$ for $-\theta_{0}<\theta<\theta_{0}-\pi$; so that we have shown that

$$
\frac{d^{2}|F(0)|}{d y^{2}}\left\{\begin{aligned}
<0 & \text { if }\left(-\pi<\theta<-\theta_{0}\right) \\
>0 & \text { if }\left(-\theta_{0}<\theta<\theta_{0}-\pi\right)
\end{aligned}\right.
$$

Since $|F(0)|=1$, then in the range $-\infty<y<\infty$, this is a maximum for $-\pi<\theta<-\theta_{0}$ and a minimum for $-\theta_{0}<\theta<\theta_{0}-\pi$. Thus we have shown that on the entire contour $|F(z)| \leq 1$ for $-\pi \leq \theta \leq-\theta_{0}$, and $|F(z)| \geq 1$ for $-\theta_{0} \leq \theta \leq \theta_{0}-\pi$. Hence by invoking the maximum modulus theorem [6] we have that $R\left(\theta, \theta_{0}\right)<1\left(-\pi<\theta<-\theta_{0}\right)$, and $R\left(\theta, \theta_{0}\right)>1\left(-\theta_{0}<\theta<\theta_{0}-\pi\right)$.

Let us denote the value of $R\left(\theta, \theta_{0}\right)$, as $y \rightarrow \infty$ by $R_{\infty}\left(\theta, \theta_{0}\right)$. Then since the largest value of $R\left(\theta, \theta_{0}\right)$, in the range $-\theta_{0}<\theta<\theta_{0}-\pi$, occurs at $y_{p}$ then we have $1<R_{\infty}\left(\theta, \theta_{0}\right)<R_{p}\left(\theta, \theta_{0}\right)$. Similarly in the range $-\pi<\theta<-\theta_{0}$, the smallest value of $R\left(\theta, \theta_{0}\right)$, occurs at $y_{p}$ and hence $1>R_{\infty}\left(\theta, \theta_{0}\right)>R_{p}\left(\theta, \theta_{0}\right)>0$.

Further it is not difficult to derive an explicit bound for $R_{\infty}\left(\theta, \theta_{0}\right)$.

$$
R_{\infty}\left(\theta, \theta_{0}\right)=\frac{\left(1-\sin \frac{1}{2} \theta-\sin \frac{1}{2} \theta_{0}\right) \cos \frac{1}{4}(\theta-\pi) \cos \frac{1}{4}\left(\theta_{0}-\pi\right)}{\left(1+\sin \frac{1}{2} \theta+\sin \frac{1}{2} \theta_{0}\right) \cos \frac{1}{4}(\theta+\pi) \cos \frac{1}{4}\left(\theta_{0}+\pi\right)}
$$




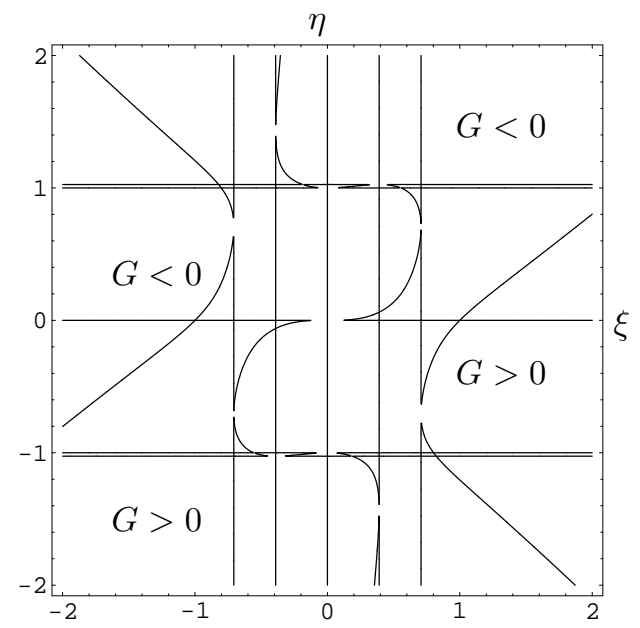

Figure 4. Regions where $G>0$ or where $G<0$.

from which we have

$$
\frac{\partial R_{\infty}\left(\theta, \theta_{0}\right)}{\partial \theta}=\frac{\left(\cos \frac{1}{4} \theta_{0}+\sin \frac{1}{4} \theta_{0}\right)\left(2-\cos \theta_{0}+\cos \theta+4 \sin \frac{1}{2} \theta_{0} \sin \frac{1}{2} \theta\right)}{4\left(\cos \frac{1}{4} \theta_{0}-\sin \frac{1}{4} \theta_{0}\right)\left(-1+\sin \frac{1}{2} \theta\right)\left(1+\sin \frac{1}{2} \theta_{0}+\sin \frac{1}{2} \theta\right)^{2}} .
$$

Thus in this range of $\theta$ the extrema occurs at those values where $\frac{\partial R_{\infty}\left(\theta, \theta_{0}\right)}{\partial \theta}=0$, that is where

$$
2-\cos \theta_{0}+\cos \theta+4 \sin \frac{1}{2} \theta_{0} \sin \frac{1}{2} \theta=0 .
$$

This last equation is a quadratic in $\sin \frac{1}{2} \theta$, that is

$$
\left(\sin \frac{1}{2} \theta\right)^{2}-2 \sin \frac{1}{2} \theta_{0}\left(\sin \frac{1}{2} \theta\right)-\frac{1}{2}\left(3-\cos \theta_{0}\right)=0,
$$

whose solutions are given by

$$
\sin \frac{1}{2} \theta=\sin \frac{1}{2} \theta_{0} \pm \sqrt{2-\cos \theta_{0}} .
$$

Clearly, since $\sin \frac{1}{2} \theta<0$, then the only relevant root is given by

$$
\theta_{\infty}=2 \arcsin \left[\sin \frac{1}{2} \theta_{0}-\sqrt{2-\cos \theta_{0}}\right] .
$$

On substituting this value into (11), we get

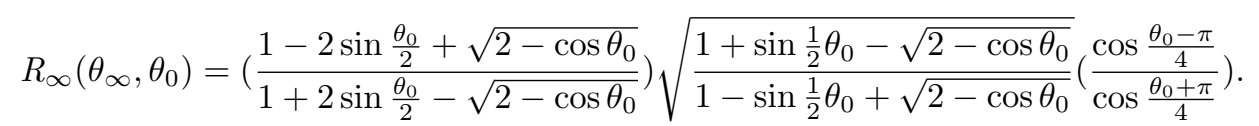

It can be seen by plotting this function with Mathematica, that it is a monotonic increasing function of $\theta_{0}$, for the range $\frac{\pi}{2}<\theta_{0}<\pi$, and $1 \leq R_{\infty}\left(\theta_{\infty}, \theta_{0}\right)<\infty$. Thus we have proved the theorem and in particular the 


\section{Corollary}

If $-\pi \leq \theta \leq \theta_{0}-\pi, \pi / 2 \leq \theta_{0} \leq \pi$, then

$$
\begin{gathered}
\left|p_{1}(r, \theta)\right| \leq\left|p_{2}(r, \theta)\right|,\left(-\pi<\theta \leq-\theta_{0}\right) ; \\
\left|p_{1}(r, \theta)\right| \geq\left|p_{2}(r, \theta)\right|,\left(-\theta_{0} \leq \theta \leq \pi-\theta_{0}\right) ;
\end{gathered}
$$

where equality occurs for $\theta=-\theta_{0}$, and $\theta=\theta_{0}-\pi$.

\section{COnClusions}

We have derived a simple and explicit expression for the ratio of acoustic pressure for different orientations of an absorbing half plane. This gives a simple analytic expression for the increase or decrease in attenuation in the shadow region of an absorbing barrier for any source $\left(\frac{\pi}{2}<\theta_{0}<\pi\right)$ - receiver $\left(-\pi<\theta<\theta_{0}-\pi\right)$ orientation. By mathematically analysing this explicit result, we have proved rigourously that in order to reduce the level of sound in the shadow region of the noise barrier the absorbing lining should be placed on the surface which makes the smallest angle from the edge of the barrier to the source or receiver; that is if $\theta_{0}<-\theta$, then the lining should be on the receiver side of the barrier, and if $\theta_{0}>-\theta$, it should be on the source side of the barrier. A physical explanation of this is that the sound waves are attenuated more effectively the nearer the ray path of the sound waves is to the absorbent surface. The present work has other interesting applications for example in mobile phone or radio wave propagation around buildings. However in this case of electromagnetic wave propagation it is often the case where one wants to increase the signal propagating round a fixed absorbing structure. The present work tells one how to achieve this for the present geometry, by positioning the source and receiver appropriately. It also will give quantitative information about the increase or decrease of the signal strength for various source receiver positions. Another aspect of the present work was the result that the magnitude of the diffracted field was the same for both half planes along the shadow boundary $\theta=\theta_{0}-\pi$. This was a surprise; and would be of some significance if it was true for any type of boundary condition on the upper and lower surfaces of the half-plane. It would be an invariant of half plane or even wedge problems along the shadow boundary and would be of some importance in inverse scattering problems.

Some interesting questions that arise from this work are : what difference would it make if the half-plane was replaced by a wedge where one face is absorbing and the other rigid? It is the author's opinion that the conclusions would not be different from above. Another question is: what difference would it make if both faces of a wedge were lined with different absorbing surfaces? This is not obvious to answer, and is mathematically more complicated. However the present method should in principle be able to deal with these cases.It is hoped to analyse, and give definitive answers, to these problems in the future. Finally the method we have devised for proving that the trigonometric expression $G$ has one sign in a certain region has potential for being a very useful tool in analyzing trigonometric inequalities. The fact that most reasonable functions can be represented by Fourier series offers even greater potential use of this method.

\section{REFERENCES}

[1] A D Rawlins. The solution of a mixed boundary value problem in the theory of diffraction by a semi-infinite plane. Proc. R. Soc. Lond. A. 346,1975,469-484. 
[2] A D Rawlins. The solution of a mixed boundary value problem in the theory of diffraction. Jour. of Eng. Maths. 18,1984,37-62.

[3] A V Osipov and $\mathrm{K}$ Hongo. Green function of an impedance wedge.Electromagnetics. 18,1998,135-165.

[4] G D Maliuzhinets. Excitation, reflection and emission of surface waves from a wedge with given face impedances.Sov. Phys. Docklady. 3,1958,752-755.

[5] R E Collin and F J Zucker. Antenna theory.Part 2.McGraw-Hill.1969, Chapter 21,304.

[6] S G Krantz. Handbook of complex variables.Birkhauser Verlag.1999, Chapter 5,76-77.

\section{Appendix A. The Karp-Karal lemma.}

Here we prove the so called Karp-Karal lemma. The author could not find a proof of the lemma that could be cited.

\section{Karp-Karal Lemma}

A surface $\left(\theta=\theta_{s}, 0<r<\infty\right)$ on which the impedance boundary condition

$$
\frac{1}{r} \frac{\partial u}{\partial \theta}+\sin \vartheta u=0, \quad\left(\theta=\theta_{s}, 0<r<\infty\right)
$$

holds, requires that any radiation field at large distances from the origin, whose leading term is asymptotically $F(\theta) \exp (\iota k r) / \sqrt{k r}$, for large $r$ must vanish on the surface $\left(\theta=\theta_{s}, 0<r<\infty\right)$.

\section{Proof}

For $r$ large enough, say $r>r_{l}$, the far field behaves like $u=F(\theta) \exp (\iota k r) / \sqrt{k r}$, then on substituting into the impedance boundary condition

$$
\begin{gathered}
\frac{1}{r} \frac{\partial u}{\partial \theta}+\sin \vartheta u=0, \quad\left(\theta=\theta_{s}, r_{l}<r<\infty\right) \\
\text { gives on }\left(\theta=\theta_{s}, r_{l}<r<\infty\right) \\
\sin \vartheta F\left(\theta_{s}\right) / \sqrt{k r}+O(k r)^{-3 / 2}=0 .
\end{gathered}
$$

Thus for $\sin \vartheta \neq 0$, and for a range of values of $r$ that are large enough, but finite, the last equation implies that $F\left(\theta_{s}\right)=0$.

\section{Appendix B. The extreme values of a real rational polynomial.}

Here we shall find the position, and possible extreme values of a real rational polynomial whose numerator and denominator are both of the third degree. Consider the real rational polynomial

$$
y=\frac{A x^{3}+B x^{2}+C x+D}{a x^{3}+b x^{2}+c x+d} .
$$

We can write this in the equivalent form

$$
(A-a y) x^{3}+(B-b y) x^{2}+(C-c y) x+(D-d y)=0 .
$$

Then on implicitly differentiating the expression (B.2), and setting $\frac{d y}{d x}=0$, gives

$$
3(A-a y) x^{2}+2(B-b y) x+(C-c y)=0 .
$$

Multiplying the equation (B.3) by $\mathrm{x} / 3$ gives

$$
(A-a y) x^{3}+\frac{2}{3}(B-b y) x^{2}+\frac{1}{3}(C-c y) x=0 .
$$

By subtracting the equation (B.4) from the equation (B.2) we get 


$$
(B-b y) x^{2}+2(C-c y) x+3(D-d y)=0 .
$$

Solving the equations (B.3) and (B.5) for y gives

$$
y=\frac{3 A x^{2}+2 B x+C}{3 a x^{2}+2 b x+c}=\frac{B x^{2}+2 C x+3 D}{b x^{2}+2 c x+3 d} ;
$$

which, on cross multiplying the last fractions, gives the quartic equation

$$
(A b-B a) x^{4}+2(A c-a C) x^{3}+(3(A d-a D)+B c-b C) x^{2}+2(B d-b D) x+3(C d-c D)=0 ;
$$

whose roots give the location of the extrema of (B.1).

To find the equation for the corresponding extrema y we eliminate $x^{2}$ and $x$ from (B.3) and (B.5) consecutively as follows. Multiply (B.3) by (B-by) and (B.5) by $3(A-a y)$ and subtracting the resulting equations from one another gives

(B.8) $2\left[(B-b y)^{2}-3(A-a y)(C-c y)\right] x+[(B-b y)(C-c y)-9(A-a y)(D-d y)]=0$.

Multiply (B.3) by $(C-c y)$ and (B.5) by $(B-b y)$ and subtracting the resultant equations one from another gives

(B.9) $2\left[3(A-a y)(C-c y)-(B-b y)^{2}\right] x^{2}+\left[(C-c y)^{2}-3(B-b y)(D-d y)\right]=0$.

Eliminating $x$ from the equations (B.8) and (B.9) results in the quartic equation:

$$
\begin{aligned}
& \left(-3 b^{2} c^{2}+12 a c^{3}+12 b^{3} d-54 a b c d+81 a^{2} d^{2}\right) y^{4}+ \\
& \left(6 b B c^{2}-12 A c^{3}+6 b^{2} c C-36 a c^{2} C-36 b^{2} B d+54 A b c d+\right. \\
& \left.54 a B c d+54 a b C d-162 a A d^{2}-12 b^{3} D+54 a b c D-162 a^{2} d D\right) y^{3}+ \\
& \left(-3 B^{2} c^{2}-12 b B c C+36 A c^{2} C-3 b^{2} C^{2}+36 a c C^{2}+36 b B^{2} d-\right. \\
& 54 A B c d-54 A b C d-54 a B C d+81 A^{2} d^{2}+3 b^{2} B D-54 A b c D- \\
& \left.54 a B c D-54 a b C D+324 a A d D+81 a^{2} D^{2}\right) y^{2}+ \\
& \left(6 B^{2} c C+6 b B C^{2}-36 A c C^{2}-12 a C^{3}-12 B^{3} d+54 A B C d-\right. \\
& \left.36 b B^{2} D+54 A B c D+54 A b C D+54 a B C D-162 A^{2} d D-162 a A D^{2}\right) y \\
& -3 B^{2} C^{2}+12 A C^{3}+12 B^{3} D-54 A B C D+81 A^{2} D^{2}=0 .
\end{aligned}
$$

whose roots give the extreme values of (B.1).

Department of Mathematical Sciences, Brunel University, Uxbridge, Middlesex, UB8 3PH, England

E-mail address: Anthony.Rawlins@brunel.ac.uk 